\title{
Clinical and Pathological Characteristics of Soft Tissue Sarcomas: A Retrospective Study From a Developing Country
}

Talal Almas ${ }^{1}$, Muhammad Kashif Khan ${ }^{2,3}$, Muhammad Faisal Murad ${ }^{4}$, Muneeb Ullah ${ }^{4}$, Adil Shafi ${ }^{5}$, Maryam Ehtesham ${ }^{1}$, Syed Muhammad Jawad Zaidi ${ }^{6}$, Salman Hussain ${ }^{1}$, Mehwish Kaneez ${ }^{6}$

1. Internal Medicine, Royal College of Surgeons in Ireland, Dublin, IRL 2. Surgical Oncology, Federal Government Poly Clinic (Post Graduate Medical Institute), Islamabad, PAK 3. Surgical Oncology, Maroof International Hospital, Islamabad, PAK 4. General Surgery, Maroof International Hospital, Islamabad, PAK 5. General Surgery, Maroof International Hospital, Islamabad, PAK 6. Internal Medicine, Rawalpindi Medical University, Rawalpindi, PAK

Corresponding author: Muhammad Kashif Khan, kashifamc@gmail.com

\section{Abstract}

\section{Introduction}

Soft tissue sarcomas remain an exceedingly rare malignancy. While soft tissue sarcomas boast a high mortality rate, their characteristics and behavior patterns are poorly understood. This study aims to evaluate the various aspects that pertain to soft tissue sarcomas, including their histology, tumor characteristics, survival rates, and therapeutic modalities.

\section{Methods}

A retrospective study analyzing the data from 19 patients presenting over four years with a histologically confirmed diagnosis of soft tissue sarcomas was conducted. The patients were studied for various parameters, including tumor site and the particular pathological subtypes. The data obtained were analyzed using the SPSS 23.0 statistical software (IBM Corporation, Armonk, NY), and the results were then tabulated.

\section{Results}

A total of 19 patients with a confirmed diagnosis of a soft tissue sarcoma were included in the study. The mean age of the patients included was $45.32 \pm 16.88$ years. Wide local excision was the most common surgical procedure employed for the resection of these tumors. Within the cohort, the mortality rate was noted to hover at $10.52 \%$. Gastrointestinal stromal tumors were observed in $21 \%$ of the patients and were therefore the most common histological subtype. Of the patients included, $42.10 \%$ required blood transfusion during the perioperative time. Most of the tumors were noted to be intermediate grade, with high-grade tumors observed in $26.3 \%$ of the cases.

Received 08/10/2020

Review began 08/13/2020 Review ended 08/13/2020 Published 08/21/2020

\section{() Copyright 2020}

Almas et al. This is an open access article distributed under the terms of the Creative Commons Attribution License CC-BY 4.0., which permits unrestricted use, distribution, and reproduction in any medium, provided the original author and source are credited.

\section{Conclusion}

Soft tissue sarcomas remain a rare but potent cause of death in developing countries. The diversity of the tissues that they afflict renders their prompt detection a diagnostic challenge. A meticulous exploration of the various characteristics honed by soft tissue sarcomas, such as the particular histological subtype and the associated mortality rates, can better elucidate the prognosis and the eventual disease outcomes.

Categories: Internal Medicine, General Surgery, Oncology

Keywords: soft tissue sarcomas, clinical characteristics, management

\section{Introduction}

Soft tissue sarcomas (STS) refer to a rare group of heterogeneous tumors of mesenchymal origin and comprise less than $0.2 \%$ of all adult cancers [1]. Due to their predilection for evoking malignant transformations in a plethora of various tissues, STS are believed to be one of the most diverse malignancies $[1,2]$. In general, STS may involve the connective tissue of the head and neck, trunk, and limbs, as well as the retroperitoneum [2]. STS manifest a spectrum of tumor behaviour, ranging from indolent growth to widespread metastasis [3]. Pertinently, the optimal management of STS remains a conundrum for clinicians, and depends on a multitude of factors, including clinical characteristics, tumor characteristics, tumor size, and the histopathological subtype [2,4]. Due to the complexity of STS, a multimodal approach is usually followed, and the utilization of a multidisciplinary approach plays a vital role in the apt management of these tumors [5].

Retroperitoneal sarcomas (RPS) are a rare group of soft tissue malignant neoplasms that comprise merely $1 \%-2 \%$ of all solid cancers and only $10 \%-20 \%$ of all sarcomas [6-8]. These tumors classically arise in the retroperitoneum and have the potential to reach exorbitant proportions without eliciting any symptoms, 


\section{Cureus}

and thus usually present late in the disease course. When RPS present with symptoms, they are usually nonspecific and include abdominal pain, abdominal discomfort, fullness, and changes in urinary or bowel habits [6]. Complete surgical resection is the standard of care for RPS, with conflicting data on the use of adjuvant and neoadjuvant therapies [9-11]. The retroperitoneum contains multiple vital organs and critical structures, including the aorta, vena cava, head of the pancreas, and duodenum $[2,6]$.

Sarcomas generally have a poor prognosis, with a five-year overall survival rate hovering around $36 \%-58 \%$ [6]. Overall survival is impacted by various prognostic factors that include histologic subtype, grade, and completeness of tumor resection [12]. While these are established prognosticators, additional parameters, such as tumor size, transfusion requirements, and anatomical location of the tumor, are also conjectured to impact the overall survival. Since surgical resection is the mainstay of management, different patterns of resection, including the employment of complex compartmental resection, are often required and are associated with varying complication rates and postoperative outcomes [13,14]. The goal is to achieve complete resection with negative macroscopic and microscopic margins to reduce the risk of local recurrence [6]. Due to the paucity of data elucidating the outcomes of STS in developing nations such as Pakistan, there is an unmet need to analyze these parameters as they pertain to STS. The present study therefore aims to delineate these parameters.

\section{Materials And Methods}

A retrospective cross-sectional study was conducted in the department of Surgical Oncology, Maroof International Hospital, Islamabad, Pakistan. A total of 19 patients who underwent surgery for a myriad of sarcomas involving various sites from January 2016 till January 2020 were included in the study. The patients were studied for various parameters, including tumor site, histopathological subtype, tumor grade, and the type of intervention employed. Patient comorbidities and various other surgical outcomes were also evaluated. Thereafter, the distribution of the various sarcomas in these patients was tabulated. The data were then analyzed using the SPSS 23.0 software (IBM Corporation, Armonk, NY).

\section{Results}

In the present study involving 19 cases, the mean age of the study participants was $45.32 \pm 16.88$ years, with a range of 20 to 80 years. Table 1 delineates the characteristics of study participants based on their gender, marital status, and comorbidities.

\begin{tabular}{|c|c|c|c|}
\hline \multicolumn{2}{|l|}{ Parameter } & Frequency & Percentage \\
\hline \multirow{2}{*}{ Gender } & Male & 10 & $52.6 \%$ \\
\hline & Female & 9 & $47.4 \%$ \\
\hline \multirow{2}{*}{ Marital status } & Married & 15 & $78.9 \%$ \\
\hline & Unmarried & 4 & $21.1 \%$ \\
\hline \multirow{3}{*}{ Comorbidities } & Hypertension & 2 & $10.5 \%$ \\
\hline & Diabetes mellitus & 2 & $10.5 \%$ \\
\hline & Ischemic heart disease & 1 & $5.3 \%$ \\
\hline
\end{tabular}

\section{TABLE 1: Background characteristics of the study participants.}

Based on the clinical evaluation, baseline laboratory investigations, and radiological imaging, the initial diagnosis was made. A plethora of various surgical procedures were deemed apt based on the site implicated and the extent of the tumor. Wide local excision was the preferred modality of surgical intervention in 16 patients, while compartmental excision was performed in merely 3 patients. Table 2 further highlights the primary site of involvement, the closest margins, the grade of the tumor, and the type of procedure employed. 


\section{Cureus}

\begin{tabular}{|c|c|c|c|}
\hline \multicolumn{2}{|l|}{ Parameter } & Frequency & Percentages \\
\hline \multirow{6}{*}{ Primary site of tumor } & Gastrointestinal tract & 3 & $15.8 \%$ \\
\hline & Abdominal/pelvic wall & 3 & $15.8 \%$ \\
\hline & Breast & 2 & $10.5 \%$ \\
\hline & Retroperitoneal & 3 & $15.8 \%$ \\
\hline & Limbs & 3 & $15.8 \%$ \\
\hline & Others & 5 & $26.3 \%$ \\
\hline \multirow{3}{*}{ Grade of tumor } & Low & 6 & $31.6 \%$ \\
\hline & Intermediate & 8 & $42.1 \%$ \\
\hline & High & 5 & $26.3 \%$ \\
\hline \multirow{4}{*}{ Closest margin } & $1-10$ mm & 6 & $31.6 \%$ \\
\hline & $10-20 \mathrm{~mm}$ & 5 & $26.3 \%$ \\
\hline & Greater than $20 \mathrm{~mm}$ & 5 & $26.3 \%$ \\
\hline & Involved & 3 & $15.8 \%$ \\
\hline \multirow{2}{*}{ Surgical procedure } & Wide local excision & 16 & $84.2 \%$ \\
\hline & Compartmental excision & 3 & $15.8 \%$ \\
\hline
\end{tabular}

TABLE 2: A tabulation of the various parameters studied with pertinence to soft tissue sarcomas.

The particular histological subtypes of the sarcomas were also evaluated. Table 3 delineates the frequency of the various histopathological subtypes.

\begin{tabular}{|l|l|}
\hline Histopathological subtype & Frequency \\
\hline Gastrointestinal stromal tumor & 4 \\
Undifferentiated pleomorphic sarcoma & 2 \\
\hline Retroperitoneal and dedifferentiated liposarcoma & 2 \\
\hline Retroperitoneal leiomyosarcoma & 1 \\
Fibrosarcoma & 1 \\
Carcinosarcoma of uterus & 1 \\
\hline Solitary fibrous tumor (malignant) & 1 \\
\hline Endometrial stromal sarcoma & 1 \\
Neurofibrosarcoma & 1 \\
Myxoid leiomyosarcoma & 1 \\
\hline Malignant spindle cell sarcoma & 1 \\
Malignant phyllodes tumor & 1 \\
Malignant peripheral nerve sheath tumor & 1 \\
Recurrent dermatofibrosarcoma protuberans & 1 \\
\hline
\end{tabular}

TABLE 3: The frequency of the various histopathological subtypes of sarcomas. 
Imperatively, merely 2 out of the 19 patients eventually died due to recurrent and persistent disease. The postoperative outcomes of the surgical interventions performed are detailed in Table 4.

\begin{tabular}{|c|c|c|}
\hline Parameter & Frequency & Percentage \\
\hline Mortality & 2 & $10.5 \%$ \\
\hline Blood transfusion required & 8 & $42.1 \%$ \\
\hline Need for re-exploration & 2 & $10.5 \%$ \\
\hline Need for re-admission & 2 & $10.5 \%$ \\
\hline Need for chemotherapy & 4 & $21 \%$ \\
\hline Need for radiotherapy & 4 & $21 \%$ \\
\hline Median operating time (range) & & $120(60-240)$ minutes \\
\hline Median hospital stay (range) & & $3(1-6)$ days \\
\hline
\end{tabular}

TABLE 4: The postoperative outcomes of patients operated for various sarcomas.

\section{Discussion}

STS remain a rare but diverse malignancy, notably affecting a vast range of different tissues and organs [1]. Imaging modalities, such as MRI, remain pivotal in detecting soft tissue tumors. CT and standard radiographs are used to rule out other possible bone tumors or cystic lesions [3,4]. After the appropriate radiological assessment, the gold standard diagnostic investigation is the core needle or excisional biopsy [5]. Given that sarcomas are noted to elicit a multitude of non-specific symptoms, they are often detected incidentally upon physical examination or imaging [6]. Ascertainment of the particular histologic subtype is performed through the means of image-guided percutaneous core needle biopsy, preferably with a co-axial technique to minimize the risk of seeding [15].

Before the consideration of the optimal management plan for the patient, a thorough preoperative evaluation of factors such as the patient's age and comorbidity status should be performed. The overarching goal, and the most efficacious treatment modality for soft tissue tumors in general and RPS in specific, is complete resection of the tumor with negative microscopic and macroscopic margins [6]. However, an accurate pathological assessment of microscopic margins is often onerous and imprecise due to the exorbitant proportions that STS can grow to. It is therefore more pragmatic to aim for a complete macroscopical resection [16]. In certain subtypes of RPS, extensive encasement of adjacent structures is noted, which often warrants an en bloc compartmental resection approach for the excision of the tumor along with the encased structures. This usually presents a formidable challenge to surgeons owing to the proximity of the tumor to various vital organs [17].

Oncological literature vouches for the notion that the grade of the tumor remains the most imperative prognostic factor [18]. Although all types of STS manifest a spectrum of behavior, ranging from indolent to malignant, certain sarcomas have a lower metastatic potential or less aggressive behavior than the other subtypes. Other prognosticators include tumor size, depth of invasion, and tumor location, with retroperitoneal tumors often boasting a worse prognosis [3,18]. Of note, completeness of tumor resection, tumor grade, and histologic subtype are all factors that are intricately linked to the overall survival [19]. Avancés et al. reported that a high histologic grade was associated with tumor recurrence and poor survival [20]. In our data, $40 \%$ of the tumors with a high histologic grade were associated with recurrence or death. In contrast, a retrospective study observed that a high histologic grade was not associated with recurrence or poor overall survival [8]. Hassan et al. demonstrated an association between histologic subtype and overall survival, particularly outlining forbidding overall survival rates and outcomes portended by

leiomyosarcomas [16]. In concert with this notion, our study divuled only two patients who were diagnosed with leiomyosarcomas, both of whom demonstrated poor prognostic outcomes. Patients with liposarcomas and lower grade sarcomas were found to have an improved overall survival [15].

Previous studies have established that complete surgical resection is the cornerstone curative treatment for RPS [21]. To this end, Malinka et al. analyzed predictors of overall survival and disease-free survival and identified surgical resection margins as one of the most important predictors of disease-free survival [22]. In our study, all patients who received blood transfusions had undergone either pelvic or abdominal surgery. Furthermore, half of those who received blood transfusions did not develop recurrence, metastasis, or a poor survival outcome. On the other hand, $25 \%$ of the patients who received transfusions eventually developed a recurrence. Whether receiving a blood transfusion serves as a viable prognostic factor remains shrouded in 
Although surgical excision remains the cornerstone of STS management, a combination of radiotherapy and/or chemotherapy remains controversial. Despite an improvement in local control, the use of chemotherapy shows no improvement in overall survival [23]. This is in accordance with a retrospective analysis that reported no beneficial effects on survival with the use of chemotherapy; however, postoperative adjuvant radiotherapy displayed beneficial effects on overall survival for truncal sarcomas [24]. This notion is in contrast to a recent analysis of the Nationwide Clinical Oncology Database (NCOD) by Nussbaum et al. that established ameliorated survival outcomes with the uptake of radiotherapy in addition to surgery when compared to surgery alone [25]. Although surgical excision remains pivotal for portending favorable outcomes, the particular efficacy of radiotherapy and chemotherapy in the optimal management of STS remains elusive. Furthermore, background patient characteristics should also be factored into decisions pertaining to the optimal treatment regimens. There is thus an overarching need for the curation of specific guidelines that can better inform the debate on the most effective modality of management in patients with STS. Larger studies with greater sample sizes are needed in order to better elucidate the outcomes and characteristics of STS, especially as they prevail in developing nations such as Pakistan.

\section{Conclusions}

STS are a rare but important cause of cancer-related mortality in developed and developing countries alike. While surgical excision remains the cornerstone treatment modality, there is an ongoing debate on the efficacy of radiotherapy and chemotherapy in thwarting the carcinogenesis of STS. Due to their non-specific symptoms, STS can evade prompt detection, often presenting as high-grade tumors that portend grave disease outcomes. A meticulous evaluation through the means of history, physical examination, and radiological imaging, followed by a core biopsy to assess the local extent of disease, therefore remains imperative. Additional studies with larger sample sizes are needed to better delineate the characteristics and behavior patterns of these tumors in order to understand the disease prognosis more comprehensively.

\section{Additional Information \\ Disclosures}

Human subjects: All authors have confirmed that this study did not involve human participants or tissue. Animal subjects: All authors have confirmed that this study did not involve animal subjects or tissue. Conflicts of interest: In compliance with the ICMJE uniform disclosure form, all authors declare the following: Payment/services info: All authors have declared that no financial support was received from any organization for the submitted work. Financial relationships: All authors have declared that they have no financial relationships at present or within the previous three years with any organizations that might have an interest in the submitted work. Other relationships: All authors have declared that there are no other relationships or activities that could appear to have influenced the submitted work.

\section{References}

1. Ratan R, Patel SR: Chemotherapy for soft tissue sarcoma . Cancer. 2016, 122:2952-2960. 10.1002/cncr.30191

2. Blay JY: Evolution in soft tissue sarcoma . Future Oncol. 2017, 13:1-2. 10.2217/fon-2016-0497

3. Fisher C: Soft tissue sarcomas: diagnosis, classification and prognostic factors . Br J Plast Surg. 1996, 49:2733. 10.1016/s0007-1226(96)90183-6

4. Blay JY, Sleijfer S, Schöffski P, Kawai A, Brodowicz T, Demetri GD, Maki RG: International expert opinion on patient-tailored management of soft tissue sarcomas. Eur J Cancer. 2014, 50:679-689. 10.1016/j.ejca.2013.11.011

5. ESMO/European Sarcoma Network Working Group: Soft tissue and visceral sarcomas: ESMO clinical practice guidelines for diagnosis, treatment and follow-up. Ann Oncol. 2012, 23:92-99. 10.1093/annonc/mds253

6. Wang J, Grignol VP, Gronchi A, Luo C-H, Pollock RE, Tseng WW: Surgical management of retroperitoneal sarcoma and opportunities for global collaboration. Chin Clin Oncol. 2018, 7:39. 10.21037/cco.2018.07.05

7. Francis IR, Cohan RH, Varma DGK, Sondak VK: Retroperitoneal sarcomas. Cancer Imaging. 2005, 5:89-94. 10.1102/1470-7330.2005.0019

8. Garcia-Ortega DY, Villa-Zepeda O, Martinez-Said H, Cuellar-Hübbe M, Luna-Ortiz K: Oncology outcomes in retroperitoneal sarcomas: prognostic factors in a retrospective cohort study. Int J Surg. 2016, 32:45-49. 10.1016/j.ijsu.2016.06.001

9. Gronchi A, Strauss DC, Miceli R, et al.: Variability in patterns of recurrence after resection of primary retroperitoneal sarcoma (RPS): a report on 1007 patients from the multi-institutional collaborative RPS working group. Ann Surg. 2016, 263:1002-1009. 10.1097/sla.0000000000001447

10. Strauss DC, Hayes AJ, Thomas JM: Retroperitoneal tumours: review of management. Ann R Coll Surg Engl. 2011, 93:275-280. 10.1308/003588411x571944

11. Almond LM, Gronchi A, Strauss D, Jafri M, Ford S, Desai A: Neoadjuvant and adjuvant strategies in retroperitoneal sarcoma. Eur J Surg Oncol. 2018, 44:571-579. 10.1016/j.ejso.2018.02.001

12. Hogg HDJ, Manas DM, Lee D, Dildey P, Scott J, Lunec J, French JJ: Surgical outcome and patterns of recurrence for retroperitoneal sarcoma at a single centre. Ann R Coll Surg Engl. 2016, 98:192-197. 10.1308/rcsann.2016.0057

13. MacNeill AJ, Fiore M: Surgical morbidity in retroperitoneal sarcoma resection. J Surg Oncol. 2018, 117:5661. 10.1002/jso. 24902 
14. MacNeill AJ, Gronchi A, Miceli R, et al.: Postoperative morbidity after radical resection of primary retroperitoneal sarcoma: a report from the transatlantic RPS working group. Ann Surg. 2018, 267:959-964. 10.1097/sla.0000000000002250

15. Van Houdt WJ, Schrijver AM, Cohen-Hallaleh RB, et al.: Needle tract seeding following core biopsies in retroperitoneal sarcoma. Eur J Surg Oncol. 2017, 43:1740-1745. 10.1016/j.ejso.2017.06.009

16. Hassan I, Park SZ, Donohue JH, et al.: Operative management of primary retroperitoneal sarcomas: a reappraisal of an institutional experience. Ann Surg. 2004, 239:244-250. 10.1097/01.sla.0000108670.31446.54

17. Almas T, Ullah M, Ehtesham M, Akbar A, Khan MK: En bloc resection of a giant retroperitoneal liposarcoma: a surgical challenge. Cureus. 2020, 12:e8730. 10.7759/cureus.8730

18. Williams KJ, Hayes AJ: A guide to oncological management of soft tissue tumours of the abdominal wall . Hernia. 2014, 18:91-97. 10.1007/s10029-013-1156-x

19. Lewis JJ, Leung D, Woodruff JM, Brennan MF: Retroperitoneal soft-tissue sarcoma: analysis of 500 patients treated and followed at a single institution. Ann Surg. 1998, 228:355-365. 10.1097/00000658-19980900000008

20. Avancès C, Mottet N, Mahatmat A, Chapuis E, Serre I, Culine S: Prognostic factors for first recurrence in patients with retroperitoneal sarcoma. Urol Oncol. 2006, 24:94-96. 10.1016/j.urolonc.2005.09.004

21. Sarcoma Meta-analysis Collaboration: Adjuvant chemotherapy for localised resectable soft-tissue sarcoma of adults: meta-analysis of individual data. Lancet. 1997, 350:1647-1654. 10.1016/S0140-6736(97)08165-8

22. Malinka T, Nebrig M, Klein F, Pratschke J, Bahra M, Andreou A: Analysis of outcomes and predictors of long-term survival following resection for retroperitoneal sarcoma. BMC Surg. 2019, 19:61. 10.1186/s12893019-0521-9

23. Singer S, Corson JM, Demetri GD, Healey EA, Marcus K, Eberlein TJ: Prognostic factors predictive of survival for truncal and retroperitoneal soft-tissue sarcoma. Ann Surg. 1995, 221:185-195. 10.1097/00000658199502000-00009

24. Catton CN, O'Sullivan B, Kotwall C, Cummings B, Hao Y, Fornasier V: Outcome and prognosis in retroperitoneal soft tissue sarcoma. Int J Radiat Oncol Biol Phys. 1994, 29:1005-1010. 10.1016/03603016(94)90395-6

25. Nussbaum DP, Rushing CN, Lane WO, Cardona DM, Kirsch DG, Peterson BL, Blazer DG: Preoperative or postoperative radiotherapy versus surgery alone for retroperitoneal sarcoma: a case-control, propensity score-matched analysis of a nationwide clinical oncology database. Lancet Oncol. 2016, 17:966-975. 10.1016/s1470-2045(16)30050-x 